\title{
Coordination In A Fully Remote Agile Software Development: A Conceptual Study
}

\author{
Prem Prakash Dayal ${ }^{1}$, M Rajkumar ${ }^{2}$ \\ ${ }^{1}$ Research Scholar, ICFAI University Jharkhand, \\ ${ }^{2}$ Associate Professor, ICFAI University Jharkhand \\ DOI: 10.29322/IJSRP.11.06.2021.p11426 \\ http://dx.doi.org/10.29322/IJSRP.11.06.2021.p11426
}

\begin{abstract}
Coordination is an important facet of agile software development and is widely used by self-organizing agile teams. While agile manifesto emphasizes face-to-face conversation in colocated setup however post the Covid-19 pandemic, IT companies have adopted remote working as new normal and agile teams have become fully remote agile teams from co-located and distributed agile teams. The current study builds a conceptual model around coordination effectiveness by examining challenges such as work from home interference, procrastination, and ineffective communication among team members working in fully remote agile software development teams.
\end{abstract}

Index Terms- Agile, work from home, coordination, pandemic, team, agile software development

\section{INTRODUCTION}

$\mathrm{W}$ ith the advent of globalization, extreme pressure to reduce cost and utilize local resource pool to gain competitive advantage, software development has transformed from a single site to globally distributed (Herbsleb \& Moitra, 2001). However, to combat the fast-changing business needs, organizations are adopting agile principles with distributed software development(Portillo-Rodríguez et al., 2012). One of the agile principles articulated in the agile manifesto by Gartner \& Barton, (2020) articulates that face to face communication is an efficient and effective way of conveying information within a development team. Thus, agile teams are preferred to be co-located (Paulk, 2002). However, in distributed software development, agile teams are distributed and pose risks around the face to face communication and team collaboration (Korkala \& Maurer, 2014; Shrivastava \& Date, 2010; Shrivastava \& Rathod, 2015)

With the implementation of social distancing norms to counter the covid-19 pandemic challenges, the agile software development teams have now become fully remote agile teams Gartner \& Barton, (2020). This means that all team members are working from home. As working from home due to the rising impact of the covid-19 pandemic becomes the new normal, there arise specific challenges and risks that remote workers would go through. It would be interesting to understand those challenges and their impact on effectiveness. Recently, Wang et al., (2021) have understood how the remote working challenges and virtual work characteristics impact the worker's effectiveness during the current pandemic.
There are studies (Mishra et al., 2012; Rola et al., 2016) that examine the different constituents of the physical work environment on communication, collaboration, and coordination in an agile software development team. Additionally, study by Strode et al., (2012) made their research work on the concept of coordination by using a model and its effectiveness in a collocated agile software development team. Hence, while there are studies around co-located and distributed agile teams, however, to the best of our knowledge, we have not come across any studies that examines the impact on coordination in a fully remote agile team setup (all team members working from home).

The current research work is an attempt to understand the impact of a fully remote agile software development team on coordination. Below mentioned is the research question that the paper intends to address

\section{Research Question}

What are the key coordination challenges in a full remote agile software development?

\section{REVIEW OF LITERATURE}

\section{Coordination:}

Effective coordination is one among the key enablers in organizations and software development. Coordination is all about managing dependencies (Crowston, 1997).In a study carried out by (Strode, 2016), a taxonomy of dependencies in agile software development has been categorized into three areas namely knowledge, process, and resource. (Stray et al., 2019) commented in their research work that, managing these dependencies in a large-scale agile project is an essential step to achieve effective coordination. Coordination mechanisms are made up of several agile practices used in software development to manage dependencies. (Strode et al., 2012) study showed as how coordination in agile software development is achieved through the three mutually exclusive components namely synchronization, structure, and boundary spanning. Additionally, the study also highlighted on the usefulness of coordination strategy in achieving coordination effectiveness.

As working from home becomes the new normal, remote workers are facing challenges such as work from home interference, procrastination, loneliness, and ineffective communication(Wang et al., 2021). In their work, one of the propositions listed is that those employees with a higher level of job autonomy, experiencing lesser work from home interference 
were exhibiting a higher level of performance. Since agile software development is a collaborative way of working and there are dependencies like the task, resource, knowledge, etc. job autonomy is relatively low and hence we argue that in remote agile software development, coordination mechanism to manage work from home interferences is required. Additionally, due to procrastination, there is delay and hence an activity dependency arises as part of the process dependency. The coordination mechanisms to manage the activity dependency are briefed and the respective impact on coordination are proposed.

\section{Autonomy and Coordination:}

Autonomy is understood as an individual's ability to make a decision around when, where, and how for the execution of the task at hand (Lu et al., 2017). As per (Wang et al., 2021), individuals working from home having higher individual autonomy at the job experience lesser work from home interference thus experiencing better productivity at work. In the context of agile software development, team autonomy is achieved by way of a self-organizing team. Self-organizing teams are also called autonomous or empowered teams who can perform tasks by owning the discretion and authority of managing all the aspects such as planning, estimation, and assignment(Moe et al., 2008; Perlak, 2019) Agile software development teams are selforganized teams. A self-organized team enjoys autonomy as they can plan based on what, when, and how to deliver. Also, one of the principles in the agile manifesto mentions the importance that the best design, architecture, and requirements get done by way of self-organizing teams(Hazzan \& Dubinsky, 2014)

However, a self-organized autonomous agile software development team also undergoes conflicts between team autonomy and individual member autonomy (Stray et al., 2018). Hence, agile software teams struggle to self-organize and have better coordination in situations where individuals enjoyed higher self-autonomy(Moe et al., 2008). A fully remote agile software development team has all individuals working from home. Individual members of the autonomous agile software development team with lower levels of job autonomy will experience more work-home interference during the period of working from home leading to lesser performance levels. Hence, based on the understanding developed from the dependency taxonomy proposed by Strode, (2016), the remote agile software development teams will experience process dependency and have a significant impact on project coordination.
Thus, we propose the below:

Proposition 1: Higher work from home interference has a negative impact on remote agile software development project coordination

\section{Social Support and Coordination:}

Social support is about experiencing a sense of care, love and value in a social network(Taylor et al., 2004).Social support has been examined in relations one of the remote work challenges which is procrastination. Procrastination is defined as a behavioural tendency to delay decisions and self- handicap oneself in completing an assigned task on time ( Chu \& Choi, 2005). Procrastination becomes worse for individuals working from home and impacts productivity(Wang et al., 2021). As per the author, the individuals received less social support and hence had more tendency to procrastinate. For individuals working in fully remote agile teams, as understood from the dependency taxonomy by Strode, (2016), the delay in task completion due to procrastination will give rise to challenges in managing activity dependency thus impacting project coordination. Hence, we propose the below

\section{Proposition 2: Lack of social support have a negative impact on remote agile software development project coordination}

\section{Communication and Coordination:}

One of the principles advocated in the agile manifesto is the face-to-face conversation to encourage information sharing within the team (Hazzan \& Dubinsky, 2014). Communication is key to success in software development and ineffective communication leads to challenges in knowledge sharing(Ghobadi \& Mathiassen, 2016). Additionally, as per the taxonomy of dependencies by Strode, (2016), this ineffective communication may lead to knowledge dependency. Additionally, as per Wang et al., (2021) ineffective communication is caused during working from home thus impacting worker's productivity. Hence, we propose the below

Proposition 3: Higher communication challenges in remote agile software development negatively impacts project coordination 


\section{Conceptual model}

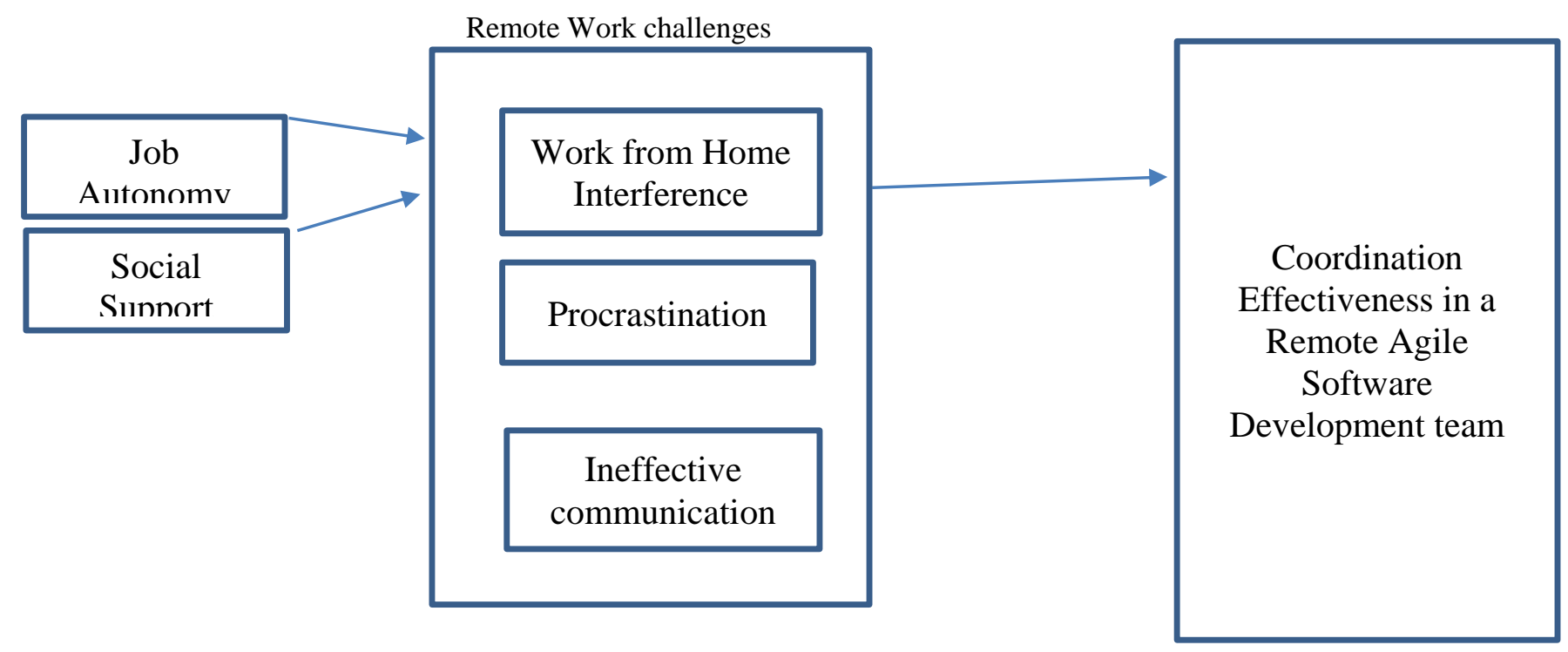

Figure 1

\section{DISCUSSION}

Agile is people-centric and several studies have examined the people-related challenges in agile software development (Gandomani et al., 2014; Gandomani \& Nafchi, 2016). Communication, collaboration, and coordination are the integral elements in software development and become more important when an agile way of working is used. Several studies have been carried out to understand the coordination mechanism around the co-located agile Strode et al., (2012) and around the distributed agile environment (Buchan et al., 2019). Similar to one of the studies conducted by Mishra et al., (2012) where the communication, coordination, and collaboration in agile software development was empirically examined in a physical work environment, this conceptual model has been built in a fully remote work environment. As the pandemic hit and people were forced to work from home, the co-located and distributed agile became fully remote agile teams.

The conceptual model in fig 1 helps to understand how coordination effectiveness is impacted in a fully remote work environment where everyone works from home. Wang et al., (2021) examined worker's performance in a virtual work characteristic with remote challenges. This conceptual model is built around the same set of virtual work characteristics and remote work challenges in the context of the fully remote agile software development team. Agile software development teams are self-organized and several studies have addressed issues and challenges around the same (Hoda et al., 2013; Hoda \& Murugesan, 2016). The proposed conceptual model also examines how coordination is impacted around the features of selforganized agile teams like autonomy and effective communication.

The first proposition considers the feature of autonomy and how the work from home interferences influence the coordination among agile team members. As agile teams are self-organized and team autonomy is a priority, the work from home interference is expected to negatively impact the coordination effectiveness among the team members. Additionally, the second proposition considers another aspect of working from home challenge where the behavioural tendency of procrastination delays the task at hand and overall coordination gets impacted. The last proposition touches upon the communication effectiveness and how challenges around communication in remote setup impacts the coordination. All the propositions are studied based on the dependency taxonomy proposed by Strode, (2016). The dependencies across knowledge, resource, and process are used while arriving at the proposition in the current conceptual model.

\section{IMPLICATIONS}

Working from home has become the new normal post the pandemic and examining agile software development around this context will have large implications both in theory and practice. To the best of our knowledge, no academic study has been carried out in the context of fully remote agile software development however considerable work has been done around co-located and distributed setup. The study also helps inform the practice about how the challenges around work from home impacts coordination and organization can adopt practices or change existing practices to enhance work from home and mitigate the risks around the same.

\section{LIMITATION OF THE PAPER}

The current research study has few limitations. The current study examines only three work from home challenges namely work from home interference, procrastination, and ineffective communication. However, other work from home challenges such as organizational climate, job-related factors, skills, and so on studied by researchers (Flores, 2019; Prasad et al., 2020) could have been considered as well. The current study considers only the 
barriers or challenges around remote work however several benefits around remote working also could have been examined. While the current conceptual model studies only one dependent variable which is coordination, future studies can also focus on well-being, collaboration, and communication as well.

\section{CONCLUSION}

Coordination is an important enabler in agile software development and a good number of studies have been carried out around the co-location (Strode et al., 2012) and distributed agile (Buchan et al., 2019). As per the recent $14^{\text {th }}$ annual agile report by Digital.ai, (2020), organizations are showing an increasing trend toward distributed teams. The current study is a small contribution in examining coordination around the context of fully remote agile software development wherein every team member works from home. The current study is built on the coordination dependency taxonomy by Strode, (2016) and identifies the dependency in the context of work from home.

\section{REFERENCES}

[1] Buchan, J., Talukder, A. B. M. N. A., \& Senapathi, M. (2019). Coordination in Distributed Agile Software Development: Insights from a COTS-based Case Study. Australasian Conference on Information Systems, 942-952.

[2] Chun Chu, A. H., \& Choi, J. N. (2005). Rethinking procrastination: Positive effects of "active" procrastination behavior on attitudes and performance. Journal of Social Psychology, 145(3), 245-264.

[3] Crowston, K. (1997). A Coordination Theory Approach to Organizational Process Design. Organization Science, 8(2), 157-175.

[4] Digital.ai. (2020). 14th annual STATE OF AGILE REPORT. Annual Report for the STATE OF AGILE, 14(14), 2-19.

[5] Flores, M. F. (2019). Understanding The Challenges Of Remote Working And It's Impact To Workers. International Journal of Business Marketing and Management (IJBMM), 4(11), 40-44.

[6] Gandomani, T. J., Zulzalil, H., Abdul Ghani, A. A., Sultan, A. B. M., \& Sharif, K. Y. (2014). How human aspects impress Agile software development transition and adoption. International Journal of Software Engineering and Its Applications, 8(1), 129-148.

[7] Gartner, \& Barton, N. (2020). Setting Up Remote Agile in a Hurry?

[8] Ghobadi, S., \& Mathiassen, L. (2016). Perceived barriers to effective knowledge sharing in agile software teams. Information Systems Journal, 26(2), 95-125.

[9] Hazzan, O., \& Dubinsky, Y. (2014). The Agile Manifesto. SpringerBriefs in Computer Science, 0(9783319101569), 9-14.

[10] Herbsleb, J. D., \& Moitra, D. (2001). Global software development. IEEE Software, 18(2), 16-20.

[11] Hoda, R., \& Murugesan, L. K. (2016). Multi-level agile project management challenges: A self-organizing team perspective. Journal of Systems and Software, 117(March), 245-257.

[12] Hoda, R., Noble, J., \& Marshall, S. (2013). Self-organizing roles on agile software development teams. IEEE Transactions on Software Engineering, 39(3), 422-444.

[13] Javdani Gandomani, T., \& Ziaei Nafchi, M. (2016). Agile transition and adoption human-related challenges and issues: A Grounded Theory approach. Computers in Human Behavior, 62, 257-266.
[14] Korkala, M., \& Maurer, F. (2014). Waste identification as the means for improving communication in globally distributed agile software development. Journal of Systems and Software, 95, 122-140.

[15] Lu, J. G., Brockner, J., Vardi, Y., \& Weitz, E. (2017). The dark side of experiencing job autonomy: Unethical behavior. Journal of Experimental Social Psychology, 73(May), 222-234.

[16] Mishra, D., Mishra, A., \& Ostrovska, S. (2012). Impact of physical ambiance on communication, collaboration and coordination in agile software development: An empirical evaluation. Information and Software Technology, 54(10), 1067-1078.

[17] Moe, N. B., Dingsøyr, T., \& Dybå, T. (2008). Understanding self-organizing teams in agile software development. Proceedings of the Australian Software Engineering Conference, ASWEC, April, 76-85.

[18] Paulk, M. C. (2002). Agile Methodologies and Process Discipline. Institute for Software Research, 15-18.

[19] Perlak, J. (2019). haracterisitcs of Self-organizing teams in Agile Project Management: A Case study. Acta Universitatis Nicolai C Opernici, 46(1), 19-27.

[20] Portillo-Rodríguez, J., Vizcaíno, A., Piattini, M., \& Beecham, S. (2012). Tools used in Global Software Engineering: A systematic mapping review. Information and Software Technology, 54(7), 663-685.

[21] Prasad, K. D. V., Mangipudi, M. R., Vaidya, R. W., \& Muralidhar, B. (2020) Organizational climate, opportunities, challenges and psychological wellbeing of the remote working employees during covid-19 pandemic: A general linear model approach with reference to information technology industry in Hyderabad. International Journal of Advanced Research in Engineering and Technology, 11(4), 372-389.

[22] Rola, P., Kuchta, D., \& Kopczyk, D. (2016). Conceptual model of working space for Agile (Scrum) project team. Journal of Systems and Software, 118, 49-63.

[23] Shrivastava, S. V., \& Date, H. (2010). Distributed Agile Software Development: A Review. 1(1), 10-17.

[24] Shrivastava, S. V., \& Rathod, U. (2015). Categorization of risk factors for distributed agile projects. Information and Software Technology, 58, $373-$ 387.

[25] Stray, V., Moe, N. B., \& Aasheim, A. (2019). Dependency Management in Large-Scale Agile: A Case Study of DevOps Teams. Proceedings of the 52nd Hawaii International Conference on System Sciences, 7007-7016.

[26] Stray, V., Moe, N. B., \& Hoda, R. (2018). Autonomous agile teams: Challenges and future directions for research. ACM International Conference Proceeding Series, Part F1477, 1-5.

[27] Strode, D. E. (2016). A dependency taxonomy for agile software development projects. Information Systems Frontiers, 18(1), 23-46.

[28] Strode, D. E., Huff, S. L., Hope, B., \& Link, S. (2012). Coordination in colocated agile software development projects. Journal of Systems and Software, 85(6), 1222-1238.

[29] Taylor, S. E., Sherman, D. K., Kim, H. S., Jarcho, J., Takagi, K., \& Dunagan, M. S. (2004). Culture and social support: Who seeks it and why? Journal of Personality and Social Psychology, 87(3), 354-362.

[30] Wang, B., Liu, Y., Qian, J., \& Parker, S. K. (2021). Achieving Effective Remote Working During the COVID-19 Pandemic: A Work Design Perspective. Applied Psychology, 70(1), 16-59.

\section{AUTHORS}

First Author - Prem Prakash Dayal, Research Scholar, ICFAI

University Jharkhand, Prem.d20@iujharkhand.edu.in

Second Author - M Rajkumar, Associate Professor, ICFAI

University Jharkhand, rajkumar.m@iujharkhand.edu.in 